\title{
Resistance-Sized Arteries Structure and Capillary Density Changes in Glioblastoma and Meningioma Peritumoral Brain Tissue
}

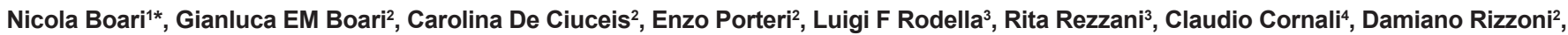 \\ Enrico Agabiti Rosei ${ }^{2}$ and Pietro Mortini ${ }^{1}$
}

${ }^{1}$ Department of Neurosurgery and Stereotactic Radiosurgery, Head and Neck Department, San Raffaele Scientific Institute, Vita-Salute University, Milan, Italy ${ }^{2}$ Clinica Medica, Department of Clinical and Experimental Sciences, University of Brescia, Brescia, Italy

${ }^{3}$ Chair of Human Anatomy, Department of Clinical and Experimental Sciences, University of Brescia, Italy

${ }^{4}$ Department of Neurosurgery, University of Brescia, Italy

\begin{abstract}
Background: Brain vessels play a relevant role in the development of malignant primary tumors. Previous studies performed in preclinical models of brain tumors demonstrated the irregular morphology of vessels in glioblastoma, characterized by multiple abnormalities in shape, permeability and relationship with the contacting structures, such as the basement membrane; however no data about microvascular structural alterations in the brain of patients affected by brain tumors have been previously reported.

Methods: We studied small vessels structural alterations, microvascular rarefaction and vascular collagen content in the peritumoral brain tissue of two groups of patients, respectively affected by glioblastoma and by meningioma, who underwent a surgical operation in order to remove the tumor. The two groups of patients were matched for clinical, laboratory variables and risk factors, with the exception of sex.

Results: Media to lumen ratio was significantly higher in patients with meningioma compared to patients affected by glioblastoma. Vessels in the brain surrounding glioblastomas show a less organized structure, as demonstrated by the thinner media, by the lower content in collagen and by the lower capillary density.

Conclusions: For the first time, we used an in vitro ex vivo technique to analyze structural alterations of resistance sized arteries of peritumoral brain tissue at the radiological tumor-brain interface in patients affected by glioblastoma and meningioma. This approach, together with immunohistochemical evaluation of microvessel density, has supplied new data about the brain microcirculation. These findings can be the result of the aberrant angiogenic process that characterizes glioblastomas and can finally play a role in tumor growth. Further studies are needed to confirm our data, to identify the pathways that lead to this microvascular pattern and to identify possible clinical applications.
\end{abstract}

Keywords: Resistance arteries; Vascular remodeling; Tumor growth; Glioblastoma; Meningioma

\section{Introduction}

Brain tumors are a heterogeneous group of both benign and malignant neoplasms affecting the central nervous system, with 12 main categories and more than 100 subcategories, with distinct biological characteristics [1].

Glioblastoma multiforme (grade IV astrocytoma) represents one of the most lethal forms of human cancer [1]. Current treatments modalities, such as surgery, chemotherapy and radiation therapy provide only modest results in terms of morbidity and mortality and the prognosis remains poor.

In the recent years, many investigations were thus performed in order to clarify the pathological process leading to tumor formation and, consequently, to identify new potential therapeutic targets [2]. These studies demonstrated that brain vessels play a relevant role in the development of malignant primary tumors, with glioblastoma being among the most angiogenic of all the tumors affecting humans [3]. Animal models provided further evidence that brain tumors rely on blood vessels for formation, growth and survival $[4,5]$.

Brain vascular system is a highly specialized structure, composed of distinct cell types forming the blood-brain barrier (BBB) [3]. Previous studies performed in preclinical models of brain tumors demonstrated the irregular morphology of vessel in glioblastoma, characterized by multiple abnormalities in shape, permeability and relationship with the contacting structures, such as the basement membrane [6].
In addition, these structural data were followed by functional analyses, which demonstrated that irregular structures support functional abnormalities, thus creating an optimal microenvironment for tumor progression [7].

However no data about microvascular structural alterations in the brain of patients with cerebral neoplasm have been previously reported. Therefore, we employed an in vitro ex vivo technique to assess structural parameters of resistance sized arteries. We also evaluated collagen content of vessel by confocal microscopy. Capillary density of excised tissue was studied by immunohistochemistry.

We evaluated two groups of patients, respectively affected by glioblastoma (astrocytoma grade IV WHO) and by meningioma (grade I WHO), in order to define and compare the structural features

*Corresponding author: Nicola Boari, M.D., Department of Neurosurgery and Gamma Knife Radiosurgery San Raffaele Scinetific Institute, Vita-Salute University, Via Olgettina 60, 20132 Milano, Italy; Fax: +39-02-26437302; Tel:+3902-26432396; E-mail: boari.nicola@hsr.it

Received February 14, 2013; Accepted March 19, 2013; Published March 22 2013

Citation: Boari N, Boari GEM, Ciuceis CD, Porteri E, Rodella LF, et al. (2013) Resistance-Sized Arteries Structure and Capillary Density Changes in Glioblastoma and Meningioma Peritumoral Brain Tissue. J Neurol Disord 1: 106. doi:10.4172/ 2329-6895.1000106

Copyright: ( 2013 Boari N, et al. This is an open-access article distributed under the terms of the Creative Commons Attribution License, which permits unrestricted use, distribution, and reproduction in any medium, provided the original author and source are credited. 
of the small resistance arteries of the brain tissue adjacent to the tumors.

\section{Patients and Methods}

\section{Patients}

We consider 7 patients with radiological and histological diagnosis of meningioma and 7 patients with radiological and histological diagnosis of glioblastoma who underwent surgical operations at the Departments of Neurosurgery of the two Centers involved in the present study. The histology of neoplasm was assessed according to WHO criteria [1]. Information about the patients was obtained by reviews of patients' clinical records, charts and imaging reports.

The two groups of patients were matched for clinical, laboratory variables and risk factors, with the exception of sex (Table 1). All patients underwent standard serum analyses, which did not reveal significant difference between the two groups (Table 1). All the patients gave their informed consent for the surgical operation and for their inclusion in the study.

Brain tissue used for our investigations was a small sample of macroscopically normal tissue obtained from the inner peritumoral area. No significant additional resection of brain tissue was performed in the patients. For the aforementioned reason Ethic Committee approval was not requested for this study.

\section{Surgery}

All the patients were operated by a standard craniotomy tailored according to the tumor location. All the surgical procedures in the group of glioblastoma patients were performed with the assistance of a navigation system using coregistration of T2 or flair and Gadolinium $\mathrm{T} 1$ images, in order to define the intraoperative radiological limit between the tumoral and peritumoral tissue.

\section{Tissue sampling}

During neurosurgical operations, a small amount of peritumoral brain tissue surrounding the tumor was sampled and rapidly put in

\begin{tabular}{|c|c|c|c|}
\hline & Meningiomas & Gliomas & p-value \\
\hline Age (years $\pm S D$ ) & $69 \pm 9$ & $6100 \mathrm{~B} 16$ & NS \\
\hline $\operatorname{Sex}(M / F)$ & $6 / 1$ & $2 / 5$ & $<0.01$ \\
\hline BMI $\left(\mathrm{m} / \mathrm{kg}^{2}\right)$ & $29 \pm 3$ & $29 \pm 3$ & NS \\
\hline Obesity & $3 / 7$ & $3 / 7$ & NS \\
\hline Hypertension & $4 / 7$ & $3 / 7$ & NS \\
\hline Hypercolesterolemia & $4 / 7$ & $4 / 7$ & NS \\
\hline Smokers & $0 / 7$ & $0 / 7$ & NS \\
\hline Systolic blood pressure $(\mathrm{mmHg} \pm \mathrm{SD})$ & $142 \pm 25$ & $132 \pm 17$ & NS \\
\hline Diastolic blood pressure $(\mathrm{mmHg} \pm \mathrm{SD})$ & $78 \pm 13$ & $78 \pm 10$ & NS \\
\hline Glycaemia (mg/DI \pm SD) & $104 \pm 16$ & $98 \pm 29$ & NS \\
\hline Cholesterolemia (mg/dL $\pm \mathrm{SD}$ ) & $164 \pm 57$ & $201 \pm 40$ & NS \\
\hline Triglycerides (mg/dL \pm SD) & $72 \pm 21$ & $123 \pm 61$ & NS \\
\hline Serum Sodium (mEq/L $\pm \mathrm{SD})$ & $142 \pm 3$ & $140 \pm 3$ & NS \\
\hline Serum Potassium (mEq/L $\pm \mathrm{SD}$ ) & $3.92 \pm 0.56$ & $4.24 \pm 0.30$ & NS \\
\hline B.U.N. ( \pm SD) & $35 \pm 14$ & $50 \pm 12$ & NS \\
\hline Serum Creatinine \pm SD) & $0.81 \pm 0.25$ & $0.99 \pm 0.26$ & NS \\
\hline Uric Acid ( \pm SD) & $3.78 \pm 2.28$ & $6.65 \pm 2.86$ & NS \\
\hline Media to lumen ratio $(M / L \pm S D)$ & $0.101 \pm 0.013$ & $0.083 \pm 0.012$ & 0.02 \\
\hline Mean collagen content ( $\%$ value $\pm \mathrm{SD})$ & $3.185 \pm 0.119$ & $1.403 \pm 0.749$ & 0.001 \\
\hline Capillary density $(X \pm S D)$ & $1.544 \pm 0.409$ & $1.157 \pm 0.330$ & 0.09 \\
\hline
\end{tabular}

Table 1: Demographic, clinical, laboratory data of patients and structural parameters of resistance-sized arteries.

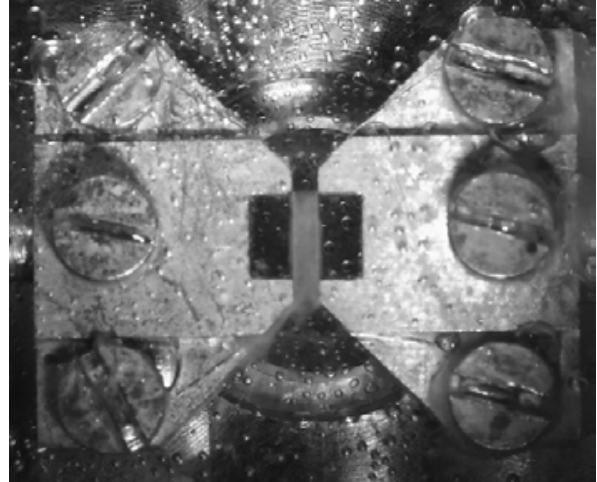

Figure 1: Vessel mounted on a wire myograph.

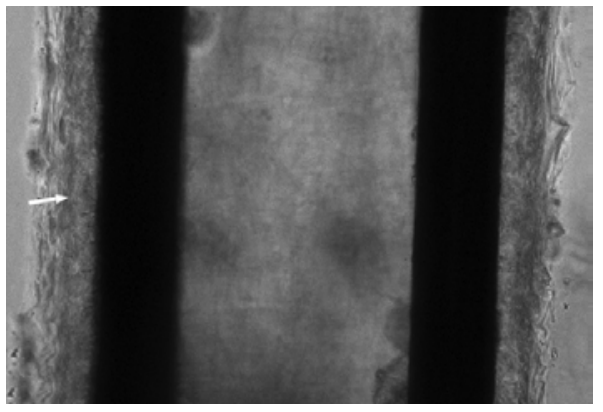

Figure 2: Vessel mounted on a wire myograph, immersion microscope: illustrative case. Arrow shows the boundary between tunica media and adventitia.

chilled physiological saline solution.

In glioblastoma operations, the sample was taken from the inner peritumoral area as assessed by pre-operative brain MRI on T2 or flair and gadolinium T1 images. In meningioma operations, a sample of brain tissue was taken by parts of the cerebrum in a non-eloquent area contused but not infiltrated by the meningiomatous mass, whose removal was necessary to reach the optimal exposure and resection of the tumor.

Cerebral small resistance arteries (about 150-350 $\mu \mathrm{m}$ of average diameter in relaxed conditions, $2 \mathrm{~mm}$ long) were dissected and mounted as a ring preparation on an isometric myograph $(410 \mathrm{~A}$, Danish Myo Technology, Aarhus, Denmark), by threading onto two stainless steel wires ( $40 \mu \mathrm{m}$ diameter) (Figure 1). After equilibration, the micromyograph was transferred to the stage of a light microscope with immersion lens. The vessel was stretched slightly (wall tension about. $0.1 \mathrm{~N} / \mathrm{m}$ ), and structural characteristics of the vessels were evaluated. The following parameters were directly measured: wall thickness, media thickness, adventitia thickness, internal diameter, media to lumen ratio and media cross-sectional area. The thicknesses of the tunica media and adventitia, and the internal diameter were measured by a micrometric ocular lens, in 6 locations ( 3 in the right side and 3 in the left side of the vessels), and the average value was considered. The boundary between the tunica media and adventitia was always clearly visible, due to the different texture of the tissues (Figure 2).

Then, the concerned morphological parameters were estimated in normalized conditions (i.e. the internal diameter the vessels would have had in vivo when subjected to a transmural pressure of $100 \mathrm{~mm} \mathrm{Hg}$ ). 
The average values obtained from two vessels in each experiment were considered. Details about the micromyographic technique of evaluation of small artery morphology were reported elsewhere [8-11].

\section{Morphological and mechanical evaluation}

In a parallel experiment, cerebral small resistance arteries $(\mathrm{n}=7$ for each group) were mounted on a pressure myograph and perfused with $\mathrm{Ca}^{+-}$free PSS containing $10 \mathrm{mmol} / \mathrm{L}$ EGTA for 30 minutes to eliminate any myogenic tone. To study vascular morphology media thickness and lumen dimension were measured at resting condition with intraluminal pressure maintained at $60 \mathrm{~mm} \mathrm{Hg}$

In order to evaluate vascular mechanics, intraluminal pressure was increased stepwise 10 to $180 \mathrm{~mm} \mathrm{Hg}$, and media and lumen dimensions were obtained at 3 different points as described elsewhere [12]. Baseline diameter was measured at $3 \mathrm{~mm} \mathrm{Hg}$.

\section{Collagen evaluation by confocal microscopy}

Cerebral small resistance arteries from 6 normotensive subjects and 6 hypertensive patients were fixed for $30 \mathrm{~min}$ under a constant intraluminal pressure of $60 \mathrm{mmHg}$ with a solution containing $3.5 \%$ formaldehyde and $0.75 \%$ glutaraldehyde in $50 \mathrm{mmol} / \mathrm{l}$ phosphate buffered saline (PBS), pH 7.4 [13]. Subsequently small arteries were treated with hyaluronidase $(1 \mathrm{mg} / \mathrm{ml})$ and incubated with antibody to collagen type I/III (1:20) (Calbiochem) overnight at $4^{\circ} \mathrm{C}$. followed by secondary antibody (Alexa Fluor 647 anti-rabbit IgG, Molecular Probes).

Rhodamine/phalloidin (Sigma, $10 \mu \mathrm{mol} / \mathrm{L}$, Molecular Probes) was added to stain $\alpha$-actin. Arteries were mounted in 1:1 glycerol/PBS (pH 7.4) on glass coverslips and studied by confocal immunofluorescence microscopy with a Zeiss LSM 510 system [14]. In order to obtain an image of the vessel as a whole, the specimen was scanned in a pointby-point to obtain a stack of sliced images (Figure 3). The amount of collagen I/III and elastin present in the vessels was quantified

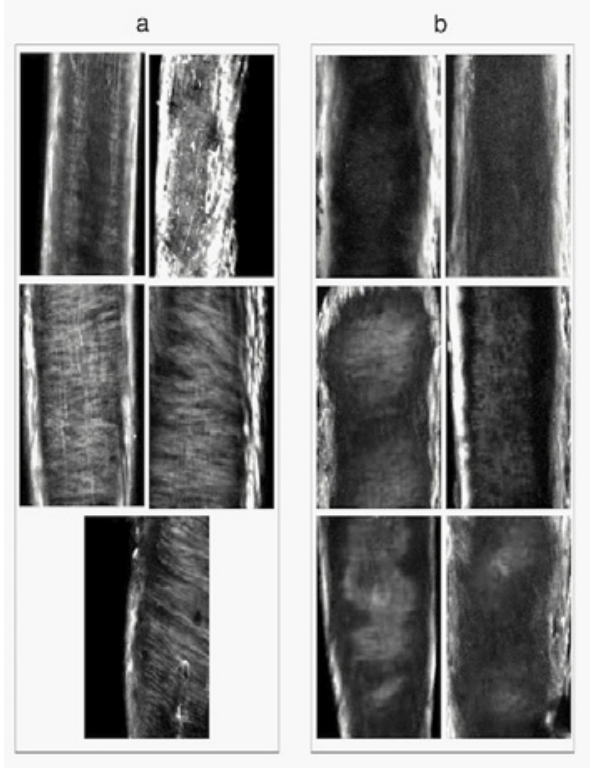

Figure 3: Confocal microscopy: illustrative cases.

(A) Collagen content in 5 cases of vessel of the brain surrounding a meningioma

(B) 6 cases of vessels of the brain surrounding a glioblastoma by imaging (Zeiss LSM Examiner Software), and expressed as a percentage of collagen/total surface area (\% pixel/surface area) $[15,16]$.

\section{Evaluation of microvessel density}

Microvessel density (MVD) was evaluated in the cerebral tissue [17]. Six frozen sections $(10 \mu \mathrm{m}$-thick) of the brain tissue were fixed in cold acetone for 10'. After fixation, sections were processed for standard immune-histochemical evaluation of the CD31. In particular, sections were incubated in $3 \%$ normal horse serum in PBS (Phosphate Buffered Saline), containing 1\% Triton, for 1 hour and then in mouse monoclonal primary antiserum directed against CD31 (Pharmigen anti-rat CD31- PECAM-1; BD Biosciences) diluted 1:100 in PBS containing 3\% normal horse serum and $1 \%$ Triton for 2 hours at $37^{\circ} \mathrm{C}$. Sections were then washed and sequentially incubated in biotinylated horse anti-mouse immunoglobulins (IgG) and avidinbiotin peroxidase complex (Vectastain $\mathrm{ABC}-\mathrm{Kit}$ ). The reaction product was visualized using $7.5 \%$ hydrogen peroxide and diaminobenzidine (DAB-2.5 mg in $10 \mathrm{ml}$ PBS) as chromogen.

Then 10 areas per sample were measured and the percentage of CD31 was calculated with an automated image analyzer (ImageproPlus, Immagini e Computer, Milan, Italy).

\section{Statistical analysis}

Statistical analysis was carried out with SPSS software (version 13.0, SPSS Inc., Chicago, Illinois, USA). All data are expressed as mean \pm SD, unless otherwise stated. Standard analyses were carried out with the use of t-test and a post-hoc Bonferroni's correction. An analysis of variance with post-hoc tests was used to evaluate differences between groups. Relationships between variables were assessed by calculation of Pearson's or Spearman's correlation coefficients according to the variable distribution. The statistical significance was set at the conventional level of 5\%. A stepwise regression analysis was performed in order to identify possible predictor of the considered parameters.

\section{Results}

Demographic characteristics of the two groups of patients (meningioma and glioblastoma) enrolled in the study are detailed in table 1 . No significant difference was detected between groups, apart for gender.

Clinical and laboratory data of patients, shown in table 1 did not show any significant difference.

\section{Microvascular morphology and density}

Results are summarized in table 1. Media to lumen ratio was significantly higher in patients with meningioma compared to patients affected by glioblastoma. No significant differences were observed in media thickness and media cross-sectional area between the two groups (data not shown).

A trend toward significance in microvascular rarefaction was observed in the cerebral tissue of patients affected by glioblastoma compared with the other group, as demonstrated by lower values of microvessel density.

Correlation analysis confirmed the correlation between presence of malignant neoplasm and $\mathrm{M} / \mathrm{L}$ ratio $(\mathrm{r}=-0.622 ; \mathrm{p}=0.018)$ as well as a borderline correlation between malignant neoplasm and capillary density $(r=-0.536 ; p=0.059)$. The regression analysis showed that the malignancy (glioblastoma) was the strongest predictor. Gender didn't show to be a predictor of the considered parameters. 


\section{Microvascular collagen}

Small cerebral arteries from patients with meningioma showed a statistically significant difference in vascular collagen deposition (expressed as percentage of total surface area) compared with those affected by glioblastoma (Table 1).

A strong correlation between presence of malignant neoplasm and collagen content was found $(\mathrm{r}=-0.853 ; \mathrm{p}=0.002)$. A weaker, but significant correlation $(\mathrm{r}=0.57 ; \mathrm{p}=0.037)$ was found with sex.

The stepwise regression analysis showed that the strongest predictor of microvascular collagen was the presence of malignant neoplasm (glioblastoma).

\section{Discussion}

Being an extra-assial tumor, meningioma, by definition, abut but do not infiltrate bordering brain tissue, thus causing brain compression leaving intact the biological features of the tissue. During surgery for meningioma removal, in particular when dealing with huge tumor, it is sometimes necessary to remove a small amount of brain tissue of non eloquent areas, compressed and macroscopically injured by the meningioma or by the surgeon during the microsurgical dissection of the tumor. We thus assumed the sample obtained during surgery for meningioma removal as biologically normal brain tissue and we compared it with the pathological counterpart represented by changes due to the presence of glioblastoma.

During glioma surgery it is sometimes difficult to discriminate the boundaries between the pathological tissue and the normal brain tissue. We consider the macroscopical limit of the tumor the radiological limit as defined on T2 or flair and gadolinium T1 MR images. To better define the intraoperative radiological tumor limit we performed all cases of glioma surgery with the support of a navigation system. However, we are also aware that the radiological limit between tumor and peritumoral area does not correspond with the biological limit of tumor infiltration. Therefore, we assumed in our study that the inner peritumoral area we sampled during glioma removal was infiltrated by the tumor $[18,19]$.

The media to lumen ratio seems to be the most reliable indicator of small resistance artery structure, because it is independent from vessels' dimension, and therefore is less affected (compared to other structural parameters such as media thickness, internal diameter or media cross-sectional area) by possible sampling bias [11].

This parameter is widely used in resistance-sized arteries structural analysis and its increment has been related to an increased risk of cardiovascular disease in hypertensive patients [20-21].

However, changes in media to lumen ratio may reflect structural changes of the media in different diseases including neoplasms and may be paradigmatic of alteration taking place during mass growth.

Another advantage of using an in vitro ex vivo technique is that we could investigate vascular collagen content, that play an important role in stabilization of wall structure.

Finally, by using an immunohistochemical technique, we were able to evaluate microvessel density. Microvasculature plays an important role in the delivery of oxygen and metabolites and is therefore critical to support tumor growth.

In our study, we observed a highly significant difference in the structures of brain vessel between glioblastoma and meningioma samples. In particular, we noted that vessels in the brain tissue surrounding glioblastomas show a less organized structure, as demonstrated by the thinner media, by the lower content in collagen and by the lower capillary density. Due to the small number of patients enrolled in this study it was not possible to exclude a role of gender, at least in vascular collagen content, although the presence of malignant neoplasm seems to be the stronger predictor. Further data are needed to clarify this point. Nevertheless the present data confirm that, at an electron microscopy in vivo study, tumor angiogenesis results from a rapid growth with tumultuous cellular proliferation leading to disorganized vessels, which are in fact diagnostic features in brain tumors $[22,23]$.

In addition, the lower capillary density observed in the present study can also lead to a heterogeneous oxygen delivery to a rapidly expanding mass, such a tumor, and eventually to a condition of hypoxia, which is typical for tumor. Hypoxia, in turn, is a condition that activates angiogenic genes, such as HIF, which further stimulate the formation of new vessels [20].

As also previously reported, the abnormal structure we observed in the present study can cause not only abnormal oxygen delivery, but also the irregular distribution of blood-borne drugs, thus limiting their efficacy for a pharmakinetics reason instead of a lack of a biological effect on the target [3].

\section{Conclusions}

For the first time, we used an in vitro ex vivo technique to analyze structural alterations of resistance sized arteries of the brain tissue adjacent to meningiomas and glioblastomas. This approach, together with immunohistochemical evaluation of microvessel density, has supplied new data about the brain microcirculation at the radiological tumor-brain interface in patients affected by glioblastoma and meningioma. In our study, we observed a highly significant difference in brain vessels structure between the two groups. In particular, we noted that vessels in the brain surrounding glioblastomas show a less organized structure, as demonstrated by the thinner media, by the lower content in collagen and by the lower capillary density. The aforementioned features can be the result of the aberrant angiogenic process that characterizes glioblastomas and can finally play a role in tumor growth. A role of gender in affecting the considered parameters cannot be excluded. Further studies in a larger group of patients are needed in order to confirm our data, to identify the pathways that lead to this microvascular pattern and to identify possible clinical applications.

\section{References}

1. Louis DN, Ohgaki H, Wiestler OD, Cavenee WK, Burger PC, et al. (2007) The 2007 WHO classification of tumours of the central nervous system. Acta Neuropathol 114: 97-109.

2. Norden AD, Drappatz J, Wen PY (2008) Novel anti-angiogenic therapies for malignant gliomas. Lancet Neurol 7: 1152-1160.

3. Jain RK, di Tomaso E, Duda DG, Loeffler JS, Sorensen AG, et al. (2007) Angiogenesis in brain tumours. Nat Rev Neurosci 8: 610-622.

4. Winkler F, Kozin SV, Tong RT, Chae SS, Booth MF, et al. (2004) Kinetics of vascular normalization by VEGFR2 blockade governs brain tumor response to radiation: role of oxygenation, angiopoietin-1, and matrix metalloproteinases. Cancer Cell 6: 553-563.

5. Fidler IJ, Yano S, Zhang RD, Fujimaki T, Bucana CD (2002) The seed and soil hypothesis: vascularisation and brain metastases. Lancet Oncol 3: 53-57.

6. Manalo DJ, Rowan A, Lavoie T, Natarajan L, Kelly BD, et al. (2005) Transcriptional regulation of vascular endothelial cell responses to hypoxia by HIF-1. Blood 105: 659-669.

7. Yuan F, Salehi HA, Boucher Y, Vasthare US, Tuma RF, et al. (1994) Vascular 
Citation: Boari N, Boari GEM, Ciuceis CD, Porteri E, Rodella LF, et al. (2013) Resistance-Sized Arteries Structure and Capillary Density Changes in

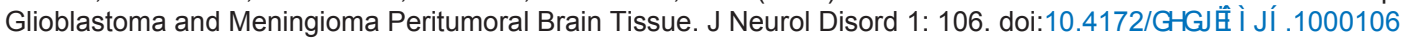

Page 5 of 5

permeability and microcirculation of gliomas and mammary carcinomas transplanted in rat and mouse cranial windows. Cancer Res 54: 4564-4568.

8. Rizzoni D, Porteri E, Castellano M, Bettoni G, Muiesan ML, et al. (1996) Vascular hypertrophy and remodeling in secondary hypertension. Hypertension 28: 785-790.

9. Aalkjaer C, Heagerty AM, Petersen KK, Swales JD, Mulvany MJ (1987) Evidence for increased media thickness, increased neural amine uptake, and depressed excitation-contraction coupling in isolated resistance vessels from essential hypertensives. Circ Res 61: 181-186.

10. Rizzoni D, Porteri E, Guelfi D, Muiesan ML, Valentini U, et al. (2001) Structura alterations in subcutaneous small arteries of normotensive and hypertensive patients with non-insulin-dependent diabetes mellitus. Circulation 103: 1238 1244.

11. Mulvany MJ, Hansen OK, Aalkjaer C (1978) Direct evidence that the greate contractility of resistance vessels in spontaneously hypertensive rats is associated with a narrowed lumen, a thickened media, and an increased number of smooth muscle cell layers. Circ Res 43: 854-864.

12. Schiffrin EL, Deng LY (1996) Structure and function of resistance arteries of hypertensive patients treated with a beta-blocker or a calcium channel antagonist. J Hypertens 14: 1247-1255.

13. Savoia C, Touyz RM, Volpe M, Schiffrin EL (2007) Angiotensin type 2 receptor in resistance arteries of type 2 diabetic hypertensive patients. Hypertension 49: $341-346$

14. Neves MF, Endemann D, Amiri F, Virdis A, Pu Q, et al. (2004) Small artery mechanics in hyperhomocysteinemic mice: effects of angiotensin II. J Hypertens 22: 959-966.
15. Virdis A, Neves MF, Amiri F, Touyz RM, Schiffrin EL (2004) Role of NAD(P) $\mathrm{H}$ oxidase on vascular alterations in angiotensin II-infused mice. J Hypertens 22: 535-542.

16. Pu Q, Neves MF, Virdis A, Touyz RM, Schiffrin EL (2003) Endothelin antagonism on aldosterone-induced oxidative stress and vascular remodeling. Hypertension 42: 49-55.

17. Munzenmaier DH, Greene AS (2006) Chronic angiotensin II AT1 recepto blockade increases cerebral cortical microvessel density. Am J Physiol Heart Circ Physiol 290: H512-516.

18. Shimizu H, Mori O, Ohaki Y, Kamoi S, Kobayashi S, et al. (2005) Cytological interface of diffusely infiltrating astrocytoma and its marginal tissue. Brain Tumor Pathol 22: 59-74.

19. McComb RD, Burger PC (1985) Pathologic analysis of primary brain tumors. Neurol Clin 3: 711-728.

20. De Ciuceis C, Porteri E, Rizzoni D, Rizzardi N, Paiardi S, et al. (2007) Structural alterations of subcutaneous small-resistance arteries may predict major cardiovascular events in patients with hypertension. Am J Hypertens 20: 846-852.

21. Mathiassen ON, Buus NH, Larsen ML, Mulvany MJ, Christensen KL (2007) Small artery structure adapts to vasodilatation rather than to blood pressure during antihypertensive treatment. J Hypertens 25: 1027-1034.

22. Rizzoni D, Porteri E, Boari GE, De Ciuceis C, Sleiman I, et al. (2003) Prognostic significance of small-artery structure in hypertension. Circulation 108: $2230-2235$.

23. Bullitt E, Muller KE, Jung I, Lin W, Aylward S (2005) Analyzing attributes of vessel populations. Med Image Anal 9: 39-49. 